\title{
Examination and redetermination of selected specimens of Cercyon Leach 1817 (Coleoptera: Hydrophildae) in the Oregon State Arthropod Collection.
}

Alyssa Lee Suzumura

Hokkaido University, Sapporo 060-8589 Japan; asuzu5@gmail.com

Cite this work as:

Suzumura, A. L. 2019. Examination and redetermination of selected specimens of Cercyon (Coleoptera: Hydrophildae) in the Oregon State Arthropod Collection. Catalog: Oregon State Arthropod Collection. 3(3) $p 1$.

DOI: http://dx.doi.org/10.5399/osu/cat osac.3.3.4612.

Fifty four specimens of interest belonging to the genus Cercyon were examined during a visit to the Oregon State Arthropod Collection in November of 2018. These belong to 4 distinct species: Cercyon luniger, C. fimbriatus, C. littoralis and C. setulosus. Some of the specimens listed below were included in a specimen loan to the author (OSAC, 2018; 2018-11-28-001-01). All of the specimens were determined or re-determined by Suzumura during her visit.

Specimens were obtained from the determined and undetermined material.

Specimens examined:

Cercyon fimbriatus Mannerheim,1852

\begin{tabular}{|c|c|c|c|}
\hline$A C \quad 000$ & OSAC 0000206894 & OSAC 00 & 10146 \\
\hline OSAC 0000185042 & OSAC 0000206934 & OSAC 0000209986 & OSAC 0000210147 \\
\hline OSAC 0000185049 & OSAC 0000209979 & OSAC 0000209987 & OSAC 000021014 \\
\hline OSAC 0000185052 & $\overline{\text { OSAC } 0000209980}$ & $\overline{\text { OSAC } 0000209988}$ & OSAC 000021014 \\
\hline OSAC 0000185111 & OSAC 0000209981 & OSAC 0000209989 & OSAC 0000210150 \\
\hline OSAC 0000206873 & OSAC 0000209982 & OSAC 0000209990 & OSAC 0000210151 \\
\hline OSAC 0000206879 & OSAC 0000209983 & OSAC 0000210018 & OSAC 0000210152 \\
\hline OSAC 000020 & C 0000209984 & OSAC 0000210145 & AC 00002 \\
\hline
\end{tabular}

Cercyon littoralis (Gyllenhal, 1808)

OSAC 0000206563

Cercyon luniger Mannerheim, 1853

OSAC 0000206564

OSAC 0000186121

OSAC 0000186122

OSAC 0000206565

OSAC 0000186123

OSAC 0001064563

OSAC 0000186124

OSAC 0001084561

OSAC 0000186125

OSAC 0001084562

OSAC 0001064565

OSAC 0001084564

Cercyon setulosus Sharp, 1884

OSAC 0000063017

OSAC 0000063018

OSAC 0000063019

OSAC 0000063020

\section{References}

OSAC. 2018. Ougoing Loans: 2018-11-28-001-01. Catalog: Oregon State Arthropod Collection. 2 (3) p. 2. DOI: http:/ / dx.doi.org/10.5399/osu/cat osac.2.3.4545. 